PROCEEDINGS OF THE

AMERICAN MATHEMATICAL SOCIETY

Volume 140, Number 4, April 2012, Pages 1371-1385

S 0002-9939(2011)11032-3

Article electronically published on August 15, 2011

\title{
HAUSDORFF DIMENSION OF SETS OF DIVERGENCE ARISING FROM CONTINUED FRACTIONS
}

\author{
IAN SHORT
}

(Communicated by Mario Bonk)

\begin{abstract}
A complex continued fraction can be represented by a sequence of Möbius transformations in such a way that the continued fraction converges if and only if the sequence converges at the origin. The set of divergence of the sequence of Möbius transformations is equivalent to the conical limit set from Kleinian group theory, and it is closely related to the Julia set from complex dynamics. We determine the Hausdorff dimensions of sets of divergence for sequences of Möbius transformations corresponding to certain important classes of continued fractions.
\end{abstract}

\section{INTRODUCTION}

This paper is about infinite continued fractions

$$
\mathbf{K}\left(a_{n} \mid b_{n}\right)=\frac{a_{1}}{b_{1}+\frac{a_{2}}{b_{2}+\frac{a_{3}}{b_{3}+\cdots}}},
$$

where $a_{i}$ and $b_{j}$ are complex numbers, and each $a_{i} \neq 0$. We define $s_{n}(z)=a_{n} /\left(b_{n}+\right.$ $z$ ) and $S_{n}=s_{1} \circ s_{2} \circ \cdots \circ s_{n}$ (or, more briefly, $S_{n}=s_{1} s_{2} \cdots s_{n}$ ) for each $n$, so that convergence of $\mathbf{K}\left(a_{n} \mid b_{n}\right)$ (in the usual sense) is equivalent to convergence of the sequence $S_{n}(0)$ within the extended complex plane $\mathbb{C}_{\infty}$. If $S_{n}(0)$ converges to a point $p$, then, because $S_{n}(\infty)=S_{n-1}(0), S_{n}(\infty)$ also converges to $p$, and in fact with the exception of at most one point $z$, the limit of $S_{n}(z)$, if it exists, is $p$ (see Theorem 3.3). The set of divergence of $S_{n}$ is the set of points $z$ in $\mathbb{C}_{\infty}$ for which the sequence $S_{n}(z)$ diverges. Sets of divergence for general sequences of Möbius transformations have been studied in [5, 6, 14. The set of divergence is closely related to the limit set from Kleinian group theory and the Julia set from complex dynamics. Indeed, sequences such as $S_{n}$ associated with continued fractions share similar properties with sequences arising in Kleinian group theory and complex dynamics (see [1, 3]). Whilst there is a large body of literature on the Hausdorff

Received by the editors July 27, 2010 and, in revised form, January 5, 2011.

2010 Mathematics Subject Classification. Primary 37F35, 40A15; Secondary 30B70, 30F45, $51 \mathrm{~B} 10$

Key words and phrases. Continued fractions, convergence, divergence, Hausdorff dimension, Möbius transformations, sets of divergence.

The author thanks the referee for useful remarks - in particular, for suggestions which strengthened Theorem 3.4 .

(C)2011 American Mathematical Society Reverts to public domain 28 years from publication 
dimension of limit sets and Julia sets, there seems to be little known about the Hausdorff dimension of sets of divergence arising from continued fractions. We take a first step in filling this gap by calculating the dimensions of sets of divergence for three well known theorems on complex and integer valued continued fractions.

The Śleszyński-Pringsheim Theorem states that $\mathbf{K}\left(a_{n} \mid b_{n}\right)$ converges provided that $\left|b_{n}\right| \geqslant 1+\left|a_{n}\right|$ for each positive integer $n$. Our first result is a generalisation of this theorem. We denote the unit disc by $\mathbb{D}$.

Theorem 1.1. If $\left|b_{n}\right| \geqslant 1+\left|a_{n}\right|$ for $n=1,2, \ldots$ then the set of divergence of $S_{n}$ is a subset of $\mathbb{C}_{\infty} \backslash \mathbb{D}$ of Hausdorff dimension less than or equal to 1 . Furthermore, there exists such a sequence $S_{n}$ whose set of divergence is a subset of $\mathbb{C}_{\infty} \backslash \mathbb{D}$ of Hausdorff dimension 1.

Since $0 \in \mathbb{D}$ it follows immediately from Theorem 1.1 that the sequence $S_{n}(0)$ converges, and hence $\mathbf{K}\left(a_{n} \mid b_{n}\right)$ converges. Therefore Theorem 1.1 generalises the Śleszyński-Pringsheim Theorem. In fact, the Śleszyński-Pringsheim Theorem is a special case of the Hillam-Thron Theorem, and we also consider Hausdorff dimensions of sets of divergence in this more general setting.

We next turn to the Seidel-Stern Theorem, which states that if $b_{1}, b_{2}, \ldots$ are positive numbers, and $\sum_{n} b_{n}$ diverges, then $\mathbf{K}\left(1 \mid b_{n}\right)$ converges. There is also a converse to the Seidel-Stern Theorem, but that does not concern us here. We define $t_{n}(z)=1 /\left(b_{n}+z\right)$ and $T_{n}=t_{1} t_{2} \cdots t_{n}$, and retain this notation throughout the paper.

Theorem 1.2. If $b_{n} \geqslant 0$ for $n=1,2, \ldots$ and $\sum_{n} b_{n}$ diverges then the set of divergence of $T_{n}$ is a subset of $(-\infty, 0)$. Furthermore, there exists such a sequence $T_{n}$ which diverges everywhere on $(-\infty, 0)$.

Theorem 1.2 contains the Seidel-Stern Theorem because 0 lies outside the set of divergence of $T_{n}$. The first part of Theorem 1.2 is known (see, for example, 4, Theorem 1.8 (ii)]).

Our final result is about continued fractions $\mathbf{K}\left(1 \mid b_{n}\right)$ in which the $b_{n}$ are positive integers. By the Seidel-Stern Theorem, all such continued fractions converge. The usual Hausdorff dimension is not sensitive enough for sets of divergence associated with these continued fractions, so instead we use logarithmic Hausdorff dimension, which is defined in Section 2. Note that a set with finite logarithmic Hausdorff dimension has (usual) Hausdorff dimension 0.

Theorem 1.3. If $b_{1}, b_{2}, \ldots$ are positive integers then the set of divergence of $T_{n}$ is a subset of $(-\infty,-1)$ of logarithmic Hausdorff dimension less than or equal to 1. Furthermore, there exists such a sequence $T_{n}$ whose set of divergence contains a closed, uncountable set within $(-\infty,-1)$.

We do not have an example of a sequence $T_{n}$ from Theorem 1.3 whose set of divergence has logarithmic Hausdorff dimension 1.

The novelty of each of these three theorems is that we obtain precise information about the sets on which our sequences of Möbius transformations converge. We make use of hyperbolic geometry and techniques borrowed from the theory of Kleinian groups. Because of this geometric approach, our results and methods generalise to higher dimensions in a straightforward fashion. 
Finally, we remark that in the metrical theory of continued fractions there is also interest in Hausdorff dimensions of sets associated with continued fractions (see, for example, [12, 17]). Typically, this theory involves the study of the set of all possible values of $\mathbf{K}\left(1 \mid b_{n}\right)$ when the coefficients $b_{n}$ are restricted in some fashion (for instance, they may be bounded positive integers). In contrast, we focus on sets of divergence associated to individual continued fractions.

\section{HAUSDORFF DIMENSION}

This section contains background information on the Hausdorff dimension, which can be found in more detail in [7, 8, 16. We use the chordal metric on $\mathbb{C}_{\infty}$ to define the Hausdorff dimension, which is the metric inherited from the Euclidean metric on the unit sphere by stereographic projection. Given a subset $X$ of $\mathbb{C}_{\infty}$, and $\varepsilon>0$, an $\varepsilon$-cover of $X$ is a collection of subsets $U_{1}, U_{2}, \ldots$ of $\mathbb{C}_{\infty}$ for which $X \subseteq \bigcup_{n} U_{n}$, and such that, for each $n$, the chordal diameter $\operatorname{diam}\left(U_{n}\right)$ of $U_{n}$ does not exceed $\varepsilon$. A dimension function is a function $f:[0,+\infty) \rightarrow[0,+\infty)$ that is increasing, continuous, and satisfies $f(0)=0$. Given a dimension function $f$ let

$$
H_{\varepsilon}^{f}(X)=\inf \left\{\sum_{n=1}^{\infty} f\left(\operatorname{diam}\left(U_{n}\right)\right): U_{1}, U_{2}, \ldots \text { is an } \varepsilon \text {-cover of } X\right\}
$$

and

$$
H^{f}(X)=\lim _{\varepsilon \rightarrow 0} H_{\varepsilon}^{f}(X)
$$

The function $H^{f}$ is known as the Hausdorff measure with respect to $f$, and it is an outer measure on $\mathbb{C}_{\infty}$.

Given two dimension functions $f$ and $g$ such that $f(t) / g(t) \rightarrow 0$ as $t \rightarrow 0$ it is straightforward to show that if $H^{g}(X)<+\infty$ then $H^{f}(X)=0$. The dimension functions most widely used are the collection $f_{s}(t)=t^{s}$, for $s>0$. Since, for $s_{1}<s_{2}, f_{s_{2}}(t) / f_{s_{1}}(t) \rightarrow 0$ as $t \rightarrow 0$, there is a unique value $d$ of $s$ in $[0,+\infty]$ such that $H^{f_{s}}(X)=+\infty$ for $s<d$, and $H^{f_{s}}(X)=0$ for $s>d$. This value $d$ is the Hausdorff dimension of $X$.

An alternative collection of dimension functions is

$$
g_{s}(t)= \begin{cases}1 /\left(\log \frac{1}{t}\right)^{s} & \text { if } 0<t \leqslant 1 / e, \\ e t & \text { otherwise }\end{cases}
$$

for $s>0$. It is only the value of $g_{s}$ near 0 that matters; the extension to $[0,+\infty)$ is chosen for convenience. Since, for $s_{1}<s_{2}, g_{s_{2}}(t) / g_{s_{1}}(t) \rightarrow 0$ as $t \rightarrow 0$, there is a unique value $d$ of $s$ in $[0,+\infty]$ such that $H^{g_{s}}(X)=+\infty$ for $s<d$, and $H^{g_{s}}(X)=0$ for $s>d$. This value $d$ is the logarithmic Hausdorff dimension of $X$. Notice that, for any positive numbers $s_{1}$ and $s_{2}, f_{s_{2}}(t) / g_{s_{1}}(t) \rightarrow 0$ as $t \rightarrow 0$, which means that if $X$ has finite logarithmic Hausdorff dimension, then it has Hausdorff dimension zero.

Let $k$ be one of the maps $f_{s}$ or $g_{s}$. Each Möbius transformation $\phi$ is a bi-Lipschitz map of $\mathbb{C}_{\infty}$ with respect to the chordal metric (see [3, Section 3]), and it follows that $H^{k}(X)=0$ if and only if $H^{k}(\phi(X))=0$. This means that $X$ and $\phi(X)$ have the same Hausdorff dimension, and they also have the same logarithmic Hausdorff dimension. 


\section{Hyperbolic GeOMETRY}

This section contains background information on hyperbolic geometry, which can be found in more detail in $\left[2, \underline{3}, 13,15\right.$. Let $\mathbb{R}_{\infty}^{3}$ denote the one-point compactification of $\mathbb{R}^{3}$. We identify $\mathbb{C}$ with the plane $x_{3}=0$ in $\mathbb{R}^{3}$ by the correspondence $x_{1}+i x_{2} \mapsto\left(x_{1}, x_{2}, 0\right)$, and we define

$$
\mathbb{H}^{3}=\left\{\left(x_{1}, x_{2}, x_{3}\right): x_{3}>0\right\},
$$

so that the closure $\overline{\mathbb{H}^{3}}$ of $\mathbb{H}^{3}$ in $\mathbb{R}_{\infty}^{3}$ is equal to $\mathbb{H}^{3} \cup \mathbb{C}_{\infty}$. We endow $\overline{\mathbb{H}^{3}}$ with the chordal metric $\chi$ which is inherited from the Euclidean metric on the closed unit ball in $\mathbb{R}^{3}$ by stereographic projection. For points $z, w \neq \infty, \chi$ is given by the formulae

$$
\chi(z, w)=\frac{2|z-w|}{\sqrt{1+|z|^{2}} \sqrt{1+|w|^{2}}}, \quad \chi(z, \infty)=\frac{2|z|}{\sqrt{1+|z|^{2}}} .
$$

The set $\mathbb{H}^{3}$ is a model of three-dimensional hyperbolic space when equipped with the Riemannian metric $|d x| / x_{3}$, and we denote the hyperbolic distance in $\mathbb{H}^{3}$ by $\varrho$. For points $x=\left(x_{1}, x_{2}, x_{3}\right)$ and $y=\left(y_{1}, y_{2}, y_{3}\right)$ in $\mathbb{H}^{3}, \varrho$ satisfies

$$
\cosh \varrho(x, y)=1+\frac{|x-y|^{2}}{2 x_{3} y_{3}} .
$$

The group $\mathcal{M}$ of Möbius transformations acts on both $\mathbb{C}_{\infty}$ and $\mathbb{H}^{3}$. It is the full group of conformal automorphisms of $\mathbb{C}_{\infty}$, and the full group of conformal hyperbolic isometries of $\mathbb{H}^{3}$. We switch freely between the two actions.

Let $j=(0,0,1)$. A sequence $F_{1}, F_{2}, \ldots$ of Möbius transformations converges generally to a point $p$ in $\mathbb{C}_{\infty}$ if the sequence $F_{n}(j)$ converges to $p$ (in the chordal metric). This terminology was introduced by Jacobsen (now Lorentzen) in 9, Definition 3.1]. Our definition is taken from [1, Theorem 3.5] and [3, Section 6], and it differs from (but is equivalent to) Lorentzen's original definition. Given another point $w$ in $\mathbb{H}^{3}$, elementary hyperbolic geometry can be used to show that $F_{n}(j)$ converges to $p$ if and only if $F_{n}(w)$ converges to $p$. The next basic lemma on general convergence, which we do not prove, follows from [3, Theorem 6.6].

Lemma 3.1. Suppose that a sequence of Möbius transformations $F_{n}$ converges at two distinct points of $\mathbb{C}_{\infty}$ to a constant $p$ in $\mathbb{C}_{\infty}$. Then $F_{n}$ converges generally to $p$.

In particular, for a convergent continued fraction with value $p$, the sequence $S_{n}$ from Section 1 converges to $p$ at both 0 and $\infty$, and hence $S_{n}$ converges generally to $p$.

Suppose that a sequence $F_{n}$ converges generally to a point $p$. Since $F_{n}$ preserves hyperbolic distance, $\varrho\left(j, F_{n}^{-1}(j)\right)=\varrho\left(F_{n}(j), j\right) \rightarrow \infty$ as $n \rightarrow \infty$. Therefore the backwards orbit $F_{n}^{-1}(j)$ accumulates only on $\mathbb{C}_{\infty}$. We describe the set of accumulation points as the Julia set of $F_{n}$, and the complement of the Julia set as the Fatou set of $F_{n}$. The Fatou set is the largest open set in $\mathbb{C}_{\infty}$ on which $F_{n}$ converges locally uniformly to $p$ [3. Theorem 9.6]. The point $j$ has no special significance in these definitions; the set of accumulation points of the backwards orbit $F_{n}^{-1}(w)$ is the same for all points $w$ in $\overline{\mathbb{H}^{3}}$ other than $p$ (see, for example, 3, Lemma 9.4]). We often use the next elementary lemma. 
Lemma 3.2. Suppose that $F_{n}$ is a generally convergent Möbius sequence and that there is a subset $X$ of $\mathbb{C}_{\infty}$ that contains at least two points, such that $F_{n}^{-1}(X) \subseteq X$ for each $n$. Then the Julia set of $F_{n}$ is contained in $\bar{X}$.

Proof. Choose a geodesic $\gamma$ that joins two distinct points $x$ and $y$ of $X$, and choose an element $w$ of $\gamma$. If $z$ is a member of the Julia set of $F_{n}$, then there is a sequence $n_{1}<n_{2}<\cdots$ such that $\chi\left(F_{n_{i}}^{-1}(w), z\right) \rightarrow 0$. This means that, given any open chordal disc $D$ in $\mathbb{C}_{\infty}$ that contains $z$, for sufficiently large $n_{i}$ the point $F_{n_{i}}^{-1}(w)$ lies in the hyperbolic convex hull of $D$ (a hyperbolic half-space). For such an $n_{i}$, one of the end-points of the geodesic $F_{n_{i}}^{-1}(\gamma)$ lies in $D$. Hence $\min \left\{\chi\left(F_{n_{i}}^{-1}(x), z\right), \chi\left(F_{n_{i}}^{-1}(y), z\right)\right\} \rightarrow 0$. Since $F_{n}^{-1}(X) \subseteq X$ for each $n$, we see that $F_{n_{i}}^{-1}(x)$ and $F_{n_{i}}^{-1}(y)$ belong to $X$. Therefore $z \in \bar{X}$.

Of more interest to us than the Julia and Fatou sets is the conical limit set of $F_{n}$, which is a subset of the Julia set. A point $z$ in $\mathbb{C}_{\infty}$ is a conical limit point of the sequence $F_{n}$ if, given a hyperbolic geodesic $\gamma$ that lands at $z$, and a point $\zeta$ in $\mathbb{H}^{3}$, there is a subsequence $w_{1}, w_{2}, \ldots$ of $F_{1}^{-1}(\zeta), F_{2}^{-1}(\zeta), \ldots$ that converges to $z$ in the chordal metric, and satisfies $\sup _{n} \varrho\left(\gamma, w_{n}\right)<+\infty$. This definition is independent of the choice of point $\zeta$ and geodesic $\gamma$. The conical limit set of $F_{n}$ consists of all its conical limit points. The next theorem indicates that for a generally convergent sequence, the conical limit set is equal to the set of divergence (with one exceptional case).

Theorem 3.3 ([1, Theorem 5.2 and Proposition 5.3]). Let $F_{n}$ be a sequence of Möbius transformations that converges generally to a point $p$. Then either (i) the conical limit set of $F_{n}$ equals the set of divergence of $F_{n}$, and $F_{n}(z)$ converges to $p$ for points $z$ outside this set, or (ii) the conical limit set consists of a single point $w$, the set of divergence is empty, and $F_{n}(z) \rightarrow p$ if and only if $z \neq w$.

The following theorem is about the Hausdorff measure of conical limit sets. We sketch the details of a proof, because although the theorem is likely to be familiar to Kleinian group theorists (it is a more general version of [13, Theorem 9.3.1]), we are unable to find a suitable reference. In this sketch of a proof (and nowhere else in the paper) we assume that the sequence $F_{n}$ is acting on the unit ball model of hyperbolic space, so that $\mathbb{C}_{\infty}$ is replaced by the unit sphere $\mathbb{S}^{2}$, the point $j$ in the statement of the theorem is replaced by 0 , and the chordal metric is replaced by the restriction of the Euclidean metric to the closed ball.

Theorem 3.4. Suppose that $g$ is a dimension function and $F_{n}$ is a generally convergent sequence of Möbius transformations such that $\sum_{n} g\left(\exp \left[-\varrho\left(j, F_{n}(j)\right)\right]\right)<+\infty$. Then the Hausdorff measure with respect to $g$ of the conical limit set of $F_{n}$ is 0 .

Proof. Let $X$ denote the conical limit set of $F_{n}$. For each point $z$ in $\mathbb{S}^{2}$ let $\gamma_{z}$ denote the geodesic half-line from 0 to $z$ (a Euclidean radius). Next, for each positive integer $m$, let $X_{m}$ consist of those points $z$ in $\mathbb{S}^{2}$ for which there is a subsequence $w_{1}, w_{2}, \ldots$ of $F_{1}^{-1}(0), F_{2}^{-1}(0), \ldots$ that converges to $z$, and satisfies $\varrho\left(\gamma_{z}, w_{n}\right)<m$ for each $n$. We must prove that $H^{g}(X)=0$, but because $X=\bigcup_{m} X_{m}$ and $H^{g}$ is countably subadditive, we need only show that $H^{g}\left(X_{m}\right)=0$ for each $m$.

Define $U_{n}=\left\{z \in \mathbb{S}^{2}: \varrho\left(\gamma_{z}, F_{n}^{-1}(0)\right)<m\right\}$ for $n=1,2, \ldots$ Observe that for any $k, U_{k}, U_{k+1}, \ldots$ is a cover of $X_{m}$. Each set $U_{n}$ is a spherical disc, and using 
hyperbolic trigonometry (see [2, Theorem 7.11.2 (ii)]) we find that for sufficiently large $n$ the Euclidean diameter $\operatorname{diam}\left(U_{n}\right)$ of $U_{n}$ satisfies

$$
\operatorname{diam}\left(U_{n}\right)=\frac{2 \sinh m}{\sinh \varrho\left(0, F_{n}^{-1}(0)\right)}<M \exp \left[-\varrho\left(0, F_{n}(0)\right)\right],
$$

where $M=8 \sinh m$. It follows from this equation that there is a positive integer $N$ such that, for each $n, U_{n}$ can be covered by $N$ spherical discs $V_{n 1}, V_{n 2}, \ldots, V_{n N}$, each of Euclidean diameter $\exp \left[-\varrho\left(0, F_{n}(0)\right)\right]$. Now

$$
\sum_{n=k}^{\infty} \sum_{i=1}^{N} g\left(\operatorname{diam}\left(V_{n i}\right)\right)=N \sum_{n=k}^{\infty} g\left(\exp \left[-\varrho\left(0, F_{n}(0)\right)\right]\right) .
$$

Since the sum on the right-hand side converges, and $\left\{V_{n i}: n \geqslant k, 1 \leqslant i \leqslant N\right\}$ is a cover of $X_{m}$ for each $k$, we see that $H^{g}\left(X_{m}\right)=0$. Thus $H^{g}(X)=0$.

We apply Theorem 3.4 first to the dimension functions $f_{s}(t)=t^{s}$ from Section 2 and then to the dimension functions $g_{s}$ from Section 2 to obtain the following two corollaries.

Corollary 3.5 ([13, Theorem 9.3.1]). Suppose that $F_{n}$ is a generally convergent sequence of Möbius transformations such that $\sum_{n} \exp \left[-s \varrho\left(j, F_{n}(j)\right)\right]<+\infty$. Then the conical limit set of $F_{n}$ has Hausdorff dimension at most $s$.

Corollary 3.6. Suppose that $F_{n}$ is a generally convergent sequence of Möbius transformations such that $\sum_{n} 1 /\left(\varrho\left(j, F_{n}(j)\right)\right)^{s}<+\infty$. Then the conical limit set of $F_{n}$ has logarithmic Hausdorff dimension at most s.

Corollary 3.5 relates to the theory of Kleinian groups. A Kleinian group $G$ is said to be of convergence type if $\sum_{g \in G} \exp [-\varrho(j, g(j))]$ converges (see [13, Section 1.6]). The critical exponent of $G$ is

$$
\inf \left\{s>0: \sum_{g \in G} \exp \left[-s \varrho\left(j, F_{n}(j)\right)\right]<+\infty\right\},
$$

and if $G$ is geometrically finite, then this equals the Hausdorff dimension of the limit set of $G$ (see [13, Theorem 9.3.6]). We do not use any of the terminology from this paragraph again.

\section{Proof of Theorem 1.1; Part I}

In this section we prove the following generalisation of the Hillam-Thron Theorem.

Theorem 4.1. Suppose that $D$ is an open disc in $\mathbb{C}_{\infty}, u$ is a point in $D, v$ is a point in $\mathbb{C}_{\infty} \backslash \bar{D}$, and $s_{1}, s_{2}, \ldots$ is a sequence of Möbius maps that satisfies $s_{n}(v)=u$ and $s_{n}(D) \subseteq D$ for each $n$. Then $S_{n}=s_{1} s_{2} \cdots s_{n}$ is generally convergent, and the conical limit set of $S_{n}$ is a subset of $\mathbb{C}_{\infty} \backslash D$ of Hausdorff dimension less than or equal to 1.

Notice that, by Theorem 3.3 , the sequence $S_{n}$ of Theorem 4.1 converges on $D$ to a constant. The usual statements of the Hillam-Thron Theorem, such as [10, Theorem 4.37], have essentially the same hypotheses as Theorem 4.1 but they have only this weaker conclusion that $S_{n}$ converges on $D$ to a constant. There are variants on the Hillam-Thron Theorem (such as [11, Lemma 3.8]) in which the 
hypothesis $s_{n}(v)=u$ is weakened, and our observations on sets of divergence apply to most, if not all, of these alternative theorems.

We now explain how the first part of Theorem 1.1 follows from Theorem 4.1] Let $s_{n}(z)=a_{n} /\left(b_{n}+z\right)$ for $n=1,2, \ldots$ It is easily shown that $\left|b_{n}\right| \geqslant 1+\left|a_{n}\right|$ if and only if $s_{n}(\mathbb{D}) \subseteq \mathbb{D}$. Thus, given a sequence $s_{1}, s_{2}, \ldots$ such that $\left|b_{n}\right| \geqslant 1+\left|a_{n}\right|$ for each $n$, the hypotheses of Theorem 4.1 are satisfied with $D=\mathbb{D}, u=0$, and $v=\infty$. Hence, by Theorem 4.1 the set of divergence of $S_{n}$ is a subset of $\mathbb{C}_{\infty} \backslash \mathbb{D}$ of Hausdorff dimension less than or equal to 1 . In fact, Theorem 1.1 is just the special case of Theorem 4.1 in which $u$ and $v$ are inverse points with respect to $\partial D$.

The key ingredient needed to move from existing statements of the Hillam-Thron Theorem to Theorem 4.1 is the following result. Recall that $j=(0,0,1)$.

Theorem 4.2. Given a sequence $F_{n}$ of Möbius transformations, suppose there are two points $x$ and $y$ in $\mathbb{C}_{\infty}$ such that $\sum_{n} \chi\left(F_{n}(x), F_{n}(y)\right)$ converges. Then $\sum_{n} \exp \left[-\varrho\left(j, F_{n}(j)\right)\right]$ also converges.

The proof of Theorem 4.2 uses the next two lemmas on hyperbolic geometry. The first is well known, so we omit the proof.

Lemma 4.3 (Theorem 7.9.1, 2]). Let $\gamma$ be a geodesic in $\mathbb{H}^{3}$ that lands at points a and $b$ in $\mathbb{C}_{\infty}$. Then

$$
\cosh \varrho(j, \gamma)=\frac{2}{\chi(a, b)} .
$$

Lemma 4.4. For a Möbius map F we have

$$
\exp [-\varrho(j, F(j))] \leqslant \frac{1}{2} \chi(F(0), F(\infty)) .
$$

Proof. Let $\gamma$ denote the geodesic between 0 and $\infty$. Since $j \in \gamma$ we have, by Lemma 4.3 .

$$
\cosh \varrho(j, F(j)) \geqslant \cosh \varrho(j, F(\gamma))=\frac{2}{\chi(F(0), F(\infty))} .
$$

The result follows because $\exp [\varrho(j, F(j))] \geqslant \cosh \varrho(j, F(j))$.

Proof of Theorem 4.2. Choose a Möbius transformation $g$ such that $g(x)=0$ and $g(y)=\infty$, and define $G_{n}=g F_{n} g^{-1}$. Since Möbius transformations are bi-Lipshitz maps of $\mathbb{C}_{\infty}$ with respect to the chordal metric, we see that $\sum_{n} \chi\left(G_{n}(0), G_{n}(\infty)\right)$ converges. Hence, by Lemma 4.4. $\sum_{n} \exp \left[-\varrho\left(j, G_{n}(j)\right)\right]$ converges. Let $u=g^{-1}(j)$. Then, by the preservation of hyperbolic distance under Möbius transformations, we deduce that $\sum_{n} \exp \left[-\varrho\left(u, F_{n}(u)\right)\right]$ converges. Since $\varrho\left(j, F_{n}(j)\right)$ differs from $\varrho\left(u, F_{n}(u)\right)$ by no more than $2 \varrho(j, u)$, we conclude that $\sum_{n} \exp \left[-\varrho\left(j, F_{n}(j)\right)\right]$ converges.

Theorem 4.1 could now be proven by modifying existing proofs of the HillamThron Theorem and then applying Theorem 4.2 however, for clarity we provide all the details in a new, complete proof which uses a mixture of hyperbolic and Euclidean geometry and which generalises easily to higher dimensions. Two lemmas are required.

Lemma 4.5. Suppose that a Euclidean circle $A$, with centre a and radius $s$, is contained within, and possibly internally tangent to, another Euclidean circle B, with centre $b$ and radius $t$. If a point $x$ that lies strictly inside $A$ is the inverse point in $A$ of another point $y$ that lies strictly inside $B$, then $|x-y| \leqslant 4(t-s)$. 
Proof. That $A$ lies inside $B$ is equivalent to the equation $|a-b|+s \leqslant t$. This inequality, together with $s \leqslant|y-a|$ and $|y-b| \leqslant t$, gives

$$
\begin{aligned}
|x-y| & =|y-a|-\frac{s^{2}}{|y-a|} \\
& =\left(1+\frac{s}{|y-a|}\right)(|y-a|-s) \\
& \leqslant 2(|y-b|+|a-b|-s) \\
& \leqslant 4(t-s) .
\end{aligned}
$$

In the next lemma we use the notation $\varrho_{D}$ for the hyperbolic metric of a disc $D$ which is strictly contained in $\mathbb{C}_{\infty}$. Notice that a Möbius transformation $f$ is an isometry from the metric space $\left(D, \varrho_{D}\right)$ to the metric space $\left(f(D), \varrho_{f(D)}\right)$.

Lemma 4.6. Let $E$ be an open Euclidean disc with centre $c$ and radius $r$. If $x$ and $y$ are two points in $E$, then

$$
|x-y|<\exp \left[\varrho_{E}(x, y)\right](r-|x-c|) .
$$

Proof. By applying a Euclidean similarity we can assume that $c=0$ and $r=1$. Next, by applying a hyperbolic rotation in $E$ about $x$, we can assume that $y$ lies on the Euclidean line $\ell$ through $x$ and 0 , in the component of $\ell \backslash\{x\}$ that contains 0 . This rotation increases the quantity $|x-y|$ but preserves $\varrho_{E}(x, y)$. Either $y$ lies between 0 and $x$ or it lies on the other side of 0 . In the first case we have

$$
\varrho_{E}(x, y)=\log \left(\frac{1+|x|}{1-|x|}\right)-\log \left(\frac{1+|y|}{1-|y|}\right)
$$

which implies that

$$
|x-y|<1-|y|=\left(\frac{1+|y|}{1+|x|}\right) \exp \left[\varrho_{E}(x, y)\right](1-|x|) \leqslant \exp \left[\varrho_{E}(x, y)\right](1-|x|) .
$$

In the second case we have

$$
\varrho_{E}(x, y)=\log \left(\frac{1+|x|}{1-|x|}\right)+\log \left(\frac{1+|y|}{1-|y|}\right),
$$

which implies that

$$
|x-y|=|x|+|y|<\frac{(1+|x|)(1+|y|)}{1-|y|}=\exp \left[\varrho_{E}(x, y)\right](1-|x|) .
$$

Proof of Theorem 4.1. Recall that the Hausdorff dimension is preserved under Möbius transformations. Therefore, after conjugating by a Möbius transformation, we may assume that $D$ is the Euclidean unit disc centred on the origin. This means that for each integer $n, S_{n}(D)$ is a Euclidean disc. Let $S_{n}(D)$ have centre $c_{n}$ and radius $r_{n}$. Since $S_{n}(D) \subseteq S_{n-1}(D)$ we obtain

$$
\left|c_{n}-c_{n-1}\right|+r_{n} \leqslant r_{n-1},
$$

from which it follows that $r_{n}$ is a decreasing (and hence convergent) sequence.

Let $v^{*}$ denote the inverse point to $v$ in $\partial D$, and let $k=\varrho_{D}\left(v^{*}, u\right)$. Inverse points are preserved under Möbius transformations, and therefore $S_{n}(v)$ and $S_{n}\left(v^{*}\right)$ are inverse points in $\partial S_{n}(D)$. Since $S_{n}(v)=S_{n-1}(u)$ lies outside $\overline{S_{n}(D)}$ but strictly 
inside $S_{n-1}(D)$, we can apply Lemma 4.5 with $A=\partial S_{n}(D), B=\partial S_{n-1}(D)$, $x=S_{n}\left(v^{*}\right)$, and $y=S_{n}(v)$ to obtain

$$
\left|S_{n}(v)-S_{n}\left(v^{*}\right)\right| \leqslant 4\left(r_{n-1}-r_{n}\right)
$$

Next, since $\varrho_{S_{n}(D)}\left(S_{n}(u), S_{n}\left(v^{*}\right)\right)=\varrho_{D}\left(u, v^{*}\right)=k$, we can apply Lemma 4.6 with $E=S_{n}(D), x=S_{n}\left(v^{*}\right)$, and $y=S_{n}(u)$ to obtain

$$
\left|S_{n}\left(v^{*}\right)-S_{n}(u)\right|<e^{k}\left(r_{n}-\left|S_{n}\left(v^{*}\right)-c_{n}\right|\right)<e^{k}\left|S_{n}(v)-S_{n}\left(v^{*}\right)\right| .
$$

Given that $S_{n}(v)=S_{n-1}(u)$, we may combine (4.2) and (4.3) using the triangle inequality to obtain

$$
\left|S_{n}(u)-S_{n-1}(u)\right| \leqslant 4\left(1+e^{k}\right)\left(r_{n-1}-r_{n}\right) .
$$

Summing this equation over all positive integers $n$ we deduce that $\sum_{n} \mid S_{n}(u)-$ $S_{n-1}(u) \mid$ converges. Since $\chi(z, w) \leqslant 2|z-w|$ for complex numbers $z$ and $w$, we see that $\sum_{n} \chi\left(S_{n}(u), S_{n-1}(u)\right)$ also converges. It follows that $S_{n}(u)$ is a Cauchy sequence which converges to a point $p$. Since $S_{n}(v)$ also converges to $p$ we see from Lemma 3.1 that $S_{n}$ converges generally to $p$. What is more, since $S_{n}^{-1}$ maps $\mathbb{C}_{\infty} \backslash D$ within itself for each $n$, we deduce from Lemma 3.2 that both the Julia set and conical limit set of $S_{n}$ are contained in $\mathbb{C}_{\infty} \backslash D$. According to Theorem 4.2, convergence of $\sum_{n} \chi\left(S_{n}(u), S_{n}(v)\right)$ implies convergence of $\sum_{n} \exp \left[-\varrho\left(j, S_{n}(j)\right)\right]$. Hence, by Corollary 3.5, the Hausdorff dimension of the conical limit set of $S_{n}$ does not exceed 1.

In fact, we have proved the slightly stronger result that the conical limit set of a sequence $S_{n}$ satisfying the hypotheses of Theorem 4.1 is a subset of $\mathbb{C}_{\infty} \backslash D$ of $H^{f_{1}}$-measure 0 .

\section{Proof of Theorem 4.1; Part II}

We must construct a sequence $s_{n}$ satisfying the hypotheses of Theorem 1.1 for which $S_{n}$ diverges on a set of Hausdorff dimension 1 . We begin by describing a suitable set of Hausdorff dimension 1. Consider a positive constant $\kappa<1 / 2$, and a null sequence of positive numbers $1=\delta_{0}, \delta_{1}, \delta_{2}, \ldots$ such that $\delta_{n} / \delta_{n-1}<\kappa$ for $n \geqslant 1$. We construct a nested sequence of sets $E_{0} \supset E_{1} \supset E_{2} \supset \cdots$ such that each set $E_{n}$ is a disjoint union of $2^{n}$ closed subintervals of $[0,1]$, each of length $\delta_{n}$. Let $E_{0}=[0,1]$. The sets $E_{1}, E_{2}, \ldots$ are defined precisely by the following rule. Each interval $[a, b]$ of $E_{n-1}$ (of length $\delta_{n-1}$ ) contains exactly two intervals of $E_{n}$, namely $\left[a, a+\delta_{n}\right]$ and $\left[b-\delta_{n}, b\right]$.

This construction is a special case of a more general construction described in [7. Example 4.6], and there it is proven that the limit set $F=\bigcap_{n} E_{n}$ is a Cantor set with Hausdorff dimension

$$
\liminf _{n \rightarrow \infty} \frac{n \log 2}{-\log \delta_{n}} .
$$

Let us choose $\delta_{n}=1 /\left(n^{2} 2^{n-1}\right)$, so that $F$ has Hausdorff dimension 1 . Let $K_{1}, K_{2}$, $\ldots$ be a sequence consisting of all the intervals from all the sets $E_{n}, n \geqslant 1$, each interval occurring only once in the sequence. If $\left|K_{i}\right|$ denotes the length of $K_{i}$ then

$$
\sum_{n=1}^{\infty}\left|K_{n}\right|=\sum_{n=1}^{\infty} 2^{n} \delta_{n}=\sum_{n=1}^{\infty} \frac{2}{n^{2}}=\frac{\pi^{2}}{3} .
$$


We scale the sets $E_{n}$ and $K_{n}$ linearly by a factor $3 / \pi^{2}$ so that now, for example, $E_{0}=\left[0,3 / \pi^{2}\right]$ and $\sum_{n}\left|K_{n}\right|=1$ (but $F$ still has Hausdorff dimension 1).

So far we have described a Cantor set of Hausdorff dimension 1 on the real line. Now we transfer that Cantor set to the unit circle $\partial \mathbb{D}$. For $n=1,2, \ldots$ let $I_{n}=\left\{e^{i t}: t \in K_{n}\right\}$, and let $\theta_{n}$ measure half the angle that $I_{n}$ makes with the origin; that is, $\theta_{n}=\frac{1}{2}\left|K_{n}\right|$. Therefore $\sum_{n} \theta_{n}=\frac{1}{2}$. Let $X$ consist of those points in $\partial \mathbb{D}$ that are contained in infinitely many of the intervals $I_{n}$. In other words, $X$ is the image of $F$ under the map from $\left[0,3 / \pi^{2}\right]$ to $\mathbb{C}$ given by $t \mapsto e^{i t}$, and since this map is bi-Lipschitz with respect to the Euclidean metric (and chordal metric), we see from [7, Corollary 2.4] that $X$ has Hausdorff dimension 1.

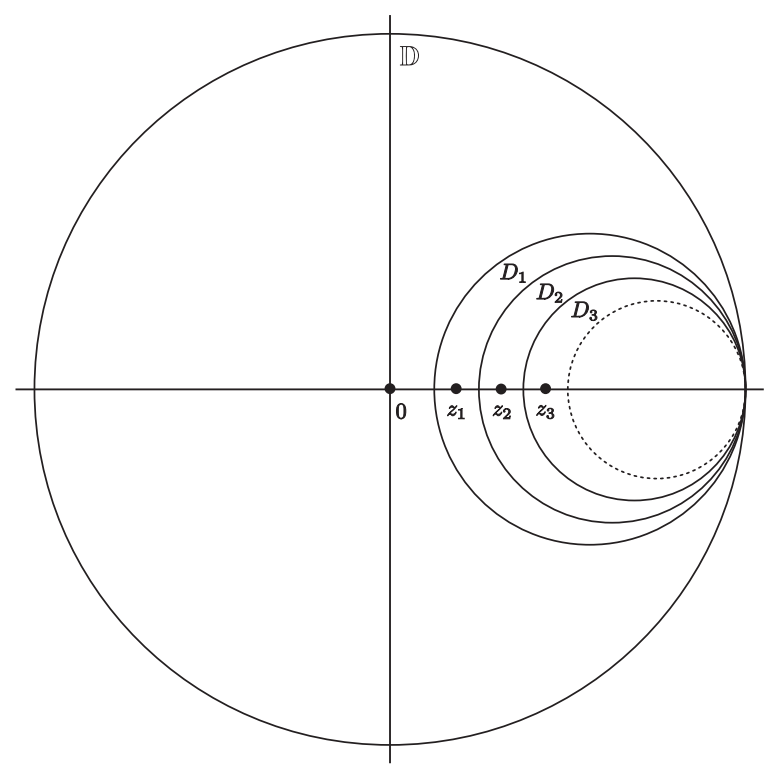

Figure 5.1

We move on to describe a sequence $S_{n}$ that diverges on this set $X$ of Hausdorff dimension 1 . Let $z_{0}=0$ and, for $n \geqslant 1$, let $z_{n}$ be the point $\theta_{1}+\cdots+\theta_{n}$ in the complex plane. Define, for $n \geqslant 1$,

$$
r_{n}=\frac{\left(1-z_{n-1}\right)\left(1-z_{n}\right)}{2-z_{n-1}-z_{n}}, \quad c_{n}=1-r_{n} .
$$

Let $D_{n}$ be the open Euclidean disc with centre $c_{n}$ and radius $r_{n}$. Then $z_{n-1}$ is exterior to $D_{n}, z_{n}$ is interior to $D_{n}$, and $z_{n-1}$ and $z_{n}$ are inverse points with respect to $D_{n}$. The points $z_{n}$ and discs $D_{n}$ are shown in Figure 5.1.

Let $S_{n}$ be a Möbius transformation which maps $\mathbb{D}$ to $D_{n}, \infty$ to $z_{n-1}$, and 0 to $z_{n}$. This is possible because 0 and $\infty$ are inverse points with respect to $\partial \mathbb{D}$, and $z_{n-1}$ and $z_{n}$ are inverse points with respect to $\partial D_{n}$. In fact there is still freedom in the choice of $S_{n}$ as we can post compose $S_{n}$ with a hyperbolic rotation of the disc $D_{n}$ about the point $z_{n}$. Thus we may further assume that $S_{n}$ maps the interval $I_{n}$ to an interval $J_{n}$ in $\partial D_{n}$ that is centred on the point 1 ( 1 is the point on $\partial D_{n}$ that is farthest from $z_{n}$ ). 
Let $J_{n}$ have end-points $a_{n}$ and $b_{n}$. Consider the hyperbolic triangle $\Delta$ with vertices $z_{n}, c_{n}$, and $a_{n}$. By conformality of $S_{n}$, the angle at $z_{n}$ of $\Delta$ is $\theta_{n}$. Let $\phi_{n}$ denote the angle of $\Delta$ at $c_{n}$. See Figure 5.2 .

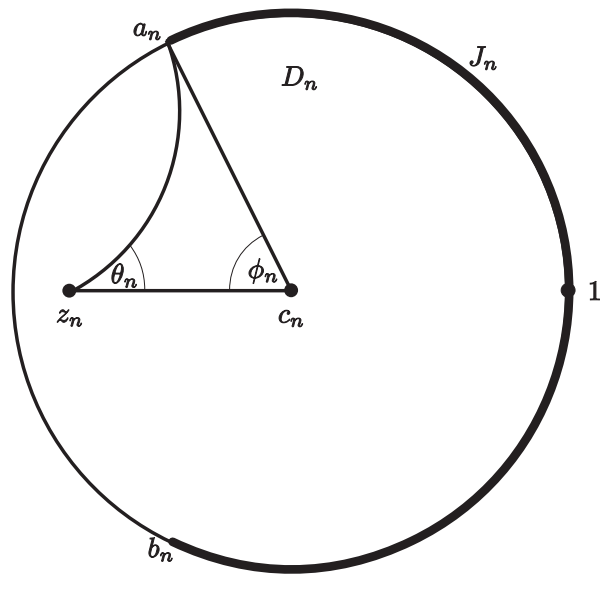

Figure 5.2

We can apply a cosine formula (see [2, Section 7.12]) to $\Delta$ to obtain

$$
\cosh \varrho_{D_{n}}\left(c_{n}, z_{n}\right)=\frac{1+\cos \theta_{n} \cos \phi_{n}}{\sin \theta_{n} \sin \phi_{n}} .
$$

Also, using a standard formula for hyperbolic distance in $D_{n}$, namely

$$
\varrho_{D_{n}}\left(c_{n}, z_{n}\right)=\log \left(\frac{r_{n}+\left|z_{n}-c_{n}\right|}{r_{n}-\left|z_{n}-c_{n}\right|}\right),
$$

we find that $\exp \left[\varrho_{D_{n}}\left(c_{n}, z_{n}\right)\right]=\left(2-z_{n}-z_{n-1}\right) / \theta_{n}$, and hence

$$
\cosh \varrho_{D_{n}}\left(c_{n}, z_{n}\right)=\frac{\left(1-z_{n}\right)^{2}+\left(1-z_{n-1}\right)^{2}}{\left(2-z_{n-1}-z_{n}\right) \theta_{n}} .
$$

Therefore

$$
1+\cos \theta_{n} \cos \phi_{n}=\frac{\left(1-z_{n}\right)^{2}+\left(1-z_{n-1}\right)^{2}}{2-z_{n-1}-z_{n}} \frac{\sin \theta_{n}}{\theta_{n}} \sin \phi_{n} .
$$

Since $\theta_{n} \rightarrow 0, z_{n} \rightarrow \frac{1}{2}$, and $\left(\sin \theta_{n}\right) / \theta_{n} \rightarrow 1$ as $n \rightarrow \infty$, we see that any limit point $\phi$ of the sequence $\phi_{n}$ is a solution of the equation

$$
2+2 \cos \phi=\sin \phi
$$

The only solutions of this equation in $[0, \pi]$ lie in $[\pi / 2, \pi]$, so there is a neighbourhood of the limit point $\frac{1}{2}$ of $z_{n}$ that intersects only finitely many of the intervals $J_{n}$. This means that if $x$ is a point that lies in infinitely many of the sets $I_{n}$, then $S_{n}(x)$ belongs to $J_{n}$ for infinitely many $n$, and $S_{n}(x) \nrightarrow \frac{1}{2}$ as $n \rightarrow \infty$. That is, $S_{n}$ does not converge to $\frac{1}{2}$ on the set $X$ of Hausdorff dimension 1 defined earlier.

It remains to verify that $S_{n}$ is of the form specified in Theorem 1.1. Define $S_{0}$ to be the identity map, and define $s_{n}=S_{n-1}^{-1} S_{n}$ for $n=1,2, \ldots$ It follows from $S_{n}(\infty)=S_{n-1}(0)$ that $s_{n}(\infty)=0$. Therefore we can write $s_{n}$ in the form $s_{n}(z)=a_{n} /\left(b_{n}+z\right)$. Next, since $S_{n}(\mathbb{D}) \subseteq S_{n-1}(\mathbb{D})$ we see that $s_{n}(\mathbb{D}) \subseteq \mathbb{D}$, and this implies that $\left|b_{n}\right| \geqslant 1+\left|a_{n}\right|$. Thus $S_{n}$ is of the required form, so it converges 
generally to $\frac{1}{2}$, and since $X$ has more than one point, we conclude, by Theorem 3.3 . that $S_{n}$ diverges on $X$.

In contrast to this example, there are sequences $s_{n}$ satisfying $\left|b_{n}\right| \geqslant 1+\left|a_{n}\right|$ for each $n$ such that $S_{n}$ converges everywhere on $\mathbb{C}_{\infty}$ to a constant. For example, suppose that $a_{n}=-1$ and $b_{n}=2$ for each $n$. Then $s_{n}(z)=-1 /(2+z)$ is a parabolic Möbius transformation with fixed point -1 , so $S_{n}$ converges everywhere to -1 .

\section{Proof of Theorem 1.2, Part I}

Let $t_{n}(z)=1 /\left(b_{n}+z\right)$ and $T_{n}=t_{1} t_{2} \cdots t_{n}$, and suppose that $b_{n} \geqslant 0$. We prove that if $\sum_{n} b_{n}$ diverges, then the set of divergence of $T_{n}$ is a subset of $(-\infty, 0)$.

The Seidel-Stern Theorem ([4, Theorem 1.8] or [11, Theorem 3.13]) states that, with our hypotheses, $T_{n}$ converges at 0 to a point $p$. Since $T_{n}(\infty)=T_{n-1}(0)$ we also have that $T_{n}(\infty) \rightarrow p$ as $n \rightarrow \infty$. Since $T_{n}$ converges to $p$ at two distinct points we see from Lemma 3.1 that $T_{n}$ converges generally to $p$. Next, observe that $t_{n}^{-1}(-\infty, 0) \subseteq(-\infty, 0)$ for each $n$ so that, by Lemma 3.2. the Julia set of $T_{n}$ is contained in $[-\infty, 0]$. Since the conical limit set is a subset of the Julia set, we see from Theorem 3.3 that the set of divergence is contained in $(-\infty, 0)$.

\section{Proof of Theorem 1.2; Part II}

In this section we construct a sequence $T_{n}$, with $b_{n} \geqslant 0$ and $\sum_{n} b_{n}=+\infty$, such that $T_{n}$ diverges everywhere on $(-\infty, 0)$.

Let $h(z)=1 / z$. For $n=1,2, \ldots$, define $g_{n}(z)=1 /(n+z)$ and $G_{n}=g_{1} g_{2} \cdots g_{n}$. Observe that $g_{n}(0,+\infty) \subseteq(0,1)$ and $g_{n}^{-1}(-\infty, 0) \subseteq(-\infty,-n)$. Then $G_{n}(0,+\infty) \subseteq$ $(0,1)$ and

$$
G_{n}^{-1}(-\infty, 0) \subseteq g_{n}^{-1}(-\infty, 0)=(-\infty,-n) .
$$

This means that $G_{n}$ is a loxodromic Möbius transformation with repelling fixed point in $[-\infty,-n]$, and attracting fixed point in $[0,1]$. Furthermore, because $G_{n}(0) \in(0,1)$ for each $n$, and using the equations $G_{n}(\infty)=G_{n-1}(0)$ and $G_{n}(-n)=$ $G_{n-1}(\infty)$, we see that neither $\infty$ nor $-n$ are fixed by $G_{n}$. Let $U_{n}$ be an open interval in $(-\infty,-n)$ that contains the repelling fixed point of $G_{n}$ and which is chosen to be small enough that $G_{n}\left(U_{n}\right) \subseteq(-\infty, 0)$. Note that $h\left(U_{n}\right) \subseteq(-1 / n, 0)$.

For each positive integer $n$, choose a positive integer $k_{n+1}$ such that the quantity $\varepsilon_{n+1}=(n+1) / k_{n+1}$ is less than half the length of the interval $h\left(U_{n}\right)$. Let $k_{1}=1$ and $\varepsilon_{1}=1$. Define $r_{n}(z)=1 /\left(z+\varepsilon_{n}\right)$. Define a sequence $t_{1}, t_{2}, \ldots$ of maps to be

$$
h, h, r_{1}, h, r_{1}, \ldots, h, r_{1}, \quad h, h, r_{2}, h, r_{2}, \ldots, h, r_{2}, \quad h, h, r_{3}, h, r_{3}, \ldots, h, r_{3}, \ldots,
$$

where the map $r_{n}$ occurs $k_{n}$ times in the above sequence. Let $T_{n}=t_{1} t_{2} \cdots t_{n}$. Observe that $g_{n}=h\left(h r_{n}\right)^{k_{n}}$. This means that $G_{n}$ is a subsequence of $T_{n}$.

Each map $t_{n}$ can be expressed uniquely in the form $t_{n}(z)=1 /\left(b_{n}+z\right)$ (where $b_{n}=\varepsilon_{m}$ if $t_{n}=r_{m}$, and $b_{n}=0$ if $\left.t_{n}=h\right)$. Clearly $b_{n} \geqslant 0$ and $\sum_{n} b_{n}$ diverges. We must show that $T_{n}$ diverges on $(-\infty, 0)$. To this end, choose a positive number $u$ (so that $-u<0$ ). We will show that $T_{n}(-u)$ diverges. Observe that, for sufficiently large $n, g_{n}(-u)>0$. Because each transformation $g_{i}$ maps $(0,+\infty)$ within itself, we deduce that $G_{n}(-u) \in G_{2}(0,+\infty)$. Thus we have found a subsequence of $T_{n}(-u)$, each element of which lies in $(2 / 3,1)$. Next we identify a subsequence of $T_{n}(-u)$ consisting of negative numbers which means that $T_{n}(-u)$ diverges. 
Consider sufficiently large values of $n$ that $1 / n<u<n$. The inequality $1 / n<u$ ensures that $-u$ lies to the left of $h\left(U_{n}\right)$ on the real axis. Therefore $u>\varepsilon_{n+1}$. Let $q_{n+1}$ be the positive integer such that $0 \leqslant u-q_{n+1} \varepsilon_{n+1}<\varepsilon_{n+1}$. Observe that

$$
q_{n+1} \leqslant \frac{u}{\varepsilon_{n+1}}<\frac{n+1}{\varepsilon_{n+1}}=k_{n+1} .
$$

Since $h\left(U_{n}\right)$ has length greater than $2 \varepsilon_{n+1}$ we can choose a positive integer $c_{n+1} \leqslant$ $q_{n+1}<k_{n+1}$ such that $-u+\varepsilon_{n+1} c_{n+1}$ lies in $h\left(U_{n}\right)$. Therefore

$$
G_{n} h\left(h r_{n+1}\right)^{c_{n+1}}(-u)=G_{n} h\left(-u+\varepsilon_{n+1} c_{n+1}\right) \in G_{n} h\left(h\left(U_{n}\right)\right)=G_{n}\left(U_{n}\right) .
$$

Since $G_{n}\left(U_{n}\right) \subseteq(-\infty, 0)$, the sequence $G_{n} h\left(h r_{n+1}\right)^{c_{n+1}}(-u)$ is a subsequence of $T_{n}(-u)$ consisting of negative numbers as required. Thus $T_{n}(-u)$ diverges.

In contrast to this example, there are sequences $t_{n}$ satisfying $\sum_{n} b_{n}=+\infty$ such that $T_{n}$ converges everywhere on $\mathbb{C}_{\infty}$ to a constant. For instance, let $t_{n}$ be the sequence $g_{n}$ from the previous example. Because $(0,+\infty) \supseteq T_{1}(0,+\infty) \supseteq$ $T_{2}(0,+\infty) \supseteq \cdots$, and $T_{n}(0)$ and $T_{n}(\infty)$ both converge to the same limit $p$, we see that $T_{n}$ converges uniformly on $[0,+\infty]$ to $p$. Given $u>0$, for sufficiently large $n$ we have $t_{n}(-u) \in[0,+\infty]$. Therefore $T_{n}(-u)$ also converges to $p$. Since $T_{n}$ also converges to $p$ away from the negative real axis, we deduce that $T_{n}$ converges to $p$ everywhere on $\mathbb{C}_{\infty}$.

\section{Proof of Theorem 1.3. Part I}

In this section we prove that the set of divergence of the sequence $T_{n}$ from Theorem 1.3 is a subset of $(-\infty,-1)$ of logarithmic Hausdorff dimension less than or equal to 1 .

The cross-ratio $[a, b, c, d]$ of four distinct points $a, b, c$, and $d$ in $\mathbb{C}_{\infty}$ is given by

$$
[a, b, c, d]=\frac{\chi(a, b) \chi(c, d)}{\chi(a, c) \chi(b, d)} .
$$

When $a, b, c$, and $d$ are all distinct from $\infty$ this equation reduces to

$$
[a, b, c, d]=\frac{|a-b||c-d|}{|a-c||b-d|} .
$$

Cross-ratios are preserved under Möbius transformations.

Lemma 8.1. We have

$$
\frac{\left|T_{n}(0)-T_{n-1}(0)\right|}{\left|T_{n-1}(0)-T_{n-2}(0)\right|}=\frac{1}{\left|b_{n} T_{n-1}^{-1}(\infty)-1\right|} .
$$

Proof. Observe that $\left[0, t_{n}(0), \infty, T_{n-1}^{-1}(\infty)\right]=\left[T_{n-1}(0), T_{n}(0), T_{n-2}(0), \infty\right]$, which follows from applying $T_{n-1}$ to the left-hand cross-ratio. The result follows immediately.

We can now prove the first part of Theorem 1.3 . Note that $t_{n}^{-1}(-\infty,-1) \subseteq$ $(-\infty,-1)$, and hence $T_{n}^{-1}(\infty) \in(-\infty,-1)$. Using Lemma 8.1 this means that

$$
\frac{\left|T_{n}(0)-T_{n-1}(0)\right|}{\left|T_{n-1}(0)-T_{n-2}(0)\right|}=\frac{1}{\left|b_{n} T_{n-1}^{-1}(\infty)-1\right|} \leqslant \frac{1}{2} \text {. }
$$

Hence

$$
\frac{1}{2} \chi\left(T_{n}(0), T_{n-1}(0)\right) \leqslant\left|T_{n}(0)-T_{n-1}(0)\right| \leqslant \frac{1}{2^{n-1}} .
$$


If we sum this equation over $n$, then we deduce that $T_{n}(0)$ is a Cauchy sequence, and hence it converges to a point $p$. Since $T_{n}(\infty)=T_{n-1}(0)$, the sequence $T_{n}(\infty)$ also converges to $p$. Therefore, by Lemma 3.1, $T_{n}$ is generally convergent to $p$. Since $t_{n}^{-1}(-\infty,-1) \subseteq(-\infty,-1)$ for each $n$ we see that, by Lemma 3.2, the Julia set of $T_{n}$ is contained in $[-\infty,-1]$. Hence $T_{n}$ converges locally uniformly to $p$ on the complement of $[-\infty,-1]$. We have already seen that $T_{n}(\infty) \rightarrow p$, and because $t_{n-1} t_{n}(-1) \in[0,1)$ for each $n$, we see that $T_{n}(-1) \rightarrow p$ also. In summary, the set of divergence of $T_{n}$ is contained in $(-\infty,-1)$, and it remains only to show that it has logarithmic Hausdorff dimension less than or equal to 1.

Using Lemma 4.4 and (8.1) we find that $\exp \left[-\varrho\left(j, T_{n}(j)\right)\right] \leqslant 1 / 2^{n-1}$, and hence, for $s>1$,

$$
\sum_{n=2}^{\infty} \frac{1}{\left(\varrho\left(j, T_{n}(j)\right)\right)^{s}} \leqslant \frac{1}{(\log 2)^{s}} \sum_{n=1}^{\infty} \frac{1}{n^{s}}<+\infty
$$

It follows from Corollary 3.6 that the logarithmic Hausdorff dimension of the conical limit set of $T_{n}$ is less than or equal to 1 . Thus, by Theorem 3.3. the logarithmic Hausdorff dimension of the set of divergence of $T_{n}$ is less than or equal to 1.

\section{Proof of Theorem 1.3: Part II}

In this section we construct a sequence of positive integers $b_{1}, b_{2}, \ldots$ such that the associated sequence of Möbius maps $T_{n}$ diverges on a closed, uncountable subset of $(-\infty,-1)$. The set we construct is a Cantor set (Cantor sets are both closed and uncountable). Let $b_{1}, b_{2}, \ldots$ be a sequence in $\{1,2\}$ with the property that each finite sequence in $\{1,2\}$ appears as a subsequence of consecutive terms in $b_{1}, b_{2}, \ldots$.

Let $I=[-3,-4 / 3]$. Define $h_{1}(z)=-1+1 / z$ and $h_{2}(z)=-2+1 / z$, and observe that $h_{1}(I)$ and $h_{2}(I)$ are disjoint subsets of $I$. Since each map $t_{n}^{-1}$ is equal to either $h_{1}$ or $h_{2}$ we see that $t_{n}^{-1}(I) \subseteq I$. Let $\Omega$ consist of those points in $I$ that lie in infinitely many of the sets $T_{n}^{-1}(I)$. If $\omega \in \Omega$, then $T_{n}(\omega) \in I$ for infinitely many $n$, which means that the sequence $T_{n}(\omega)$ does not converge to the (positive) value of the continued fraction, so it must diverge. Hence $\Omega$ is contained in the set of divergence of $T_{n}$.

Now, given any interval $T_{n}^{-1}(I)$ we can choose an integer $p$ larger than $n$ such that the first $n+1$ terms of the sequence $b_{p}, b_{p-1}, \ldots, b_{1}$ are, in order, $b_{n}, b_{n-1}, \ldots, b_{1}, 1$, in which case,

$$
T_{p}^{-1}(I)=T_{n}^{-1} h_{1} T_{p-n-1}^{-1}(I) \subseteq T_{n}^{-1}\left(h_{1}(I)\right) .
$$

Likewise we can choose $q>n$ such that $T_{q}^{-1}(I) \subseteq T_{n}^{-1}\left(h_{2}(I)\right)$. Since $h_{1}(I)$ and $h_{2}(I)$ are disjoint subintervals of $I$ it follows that $T_{p}^{-1}(I)$ and $T_{q}^{-1}(I)$ are disjoint subintervals of $T_{n}^{-1}(I)$. By repeatedly choosing pairs of subintervals in this way we see that $\Omega$, and hence the set of divergence of $T_{n}$, contains a Cantor set in $(-\infty,-1)$.

Since $\Omega$ contains a set that is both closed and uncountable, it follows from [16, Corollary 1 , Section 3] that there exists a dimension function $k$ for which $H^{k}(\Omega)>0$. We are unable to construct, for any $s \leqslant 1$, a set of divergence $X$ associated with Theorem 1.3 such that $H^{g_{s}}(X)>0$. 


\section{REFERENCES}

[1] B. Aebischer, The limiting behavior of sequences of Möbius transformations, Math. Z. 205 (1990), no. 1, 49-59. MR1069484 (91i:51007)

[2] A. F. Beardon, The geometry of discrete groups, Springer, New York, 1983. MR698777 $(85 \mathrm{~d}: 22026)$

[3] A. F. Beardon, Continued fractions, discrete groups and complex dynamics, Comput. Methods Funct. Theory 1 (2001), no. 2, 535-594. MR1941142 (2003m:30010)

[4] A. F. Beardon and I. Short, The Seidel, Stern, Stolz and Van Vleck Theorems on continued fractions, Bull. London Math. Soc. 42 (2010), no. 3, 457-466. MR2651941

[5] E. Crane and I. Short, Conical limit sets and continued fractions, Conform. Geom. Dyn. 11 (2007), 224-249 (electronic). MR2354097(2008i:30002)

[6] P. Erdős and G. Piranian, Sequences of linear fractional transformations, Michigan Math. J 6 (1959), 205-209. MR0109227 (22:114)

[7] K. Falconer, Fractal geometry, Second edition, Wiley, Hoboken, NJ, 2003. MR 2118797 (2006b:28001)

[8] W. K. Hayman and P. B. Kennedy, Subharmonic functions. Vol. I, Academic Press, London, 1976. MR0460672 (57:665)

[9] L. Jacobsen, General convergence of continued fractions, Trans. Amer. Math. Soc. 294 (1986), no. 2, 477-485. MR825716 (87j:40004)

[10] W. B. Jones and W. J. Thron, Continued fractions, Addison-Wesley Publishing Co., Reading, Mass., 1980. MR595864 (82c:30001)

[11] L. Lorentzen and H. Waadeland, Continued fractions. Vol. 1, Second edition, Atlantis Press, Paris, 2008. MR2433845 (2009b:30005)

[12] R. D. Mauldin and M. Urbański, Conformal iterated function systems with applications to the geometry of continued fractions, Trans. Amer. Math. Soc. 351 (1999), no. 12, 4995-5025. MR.1487636 (2000c:28016)

[13] P. J. Nicholls, The ergodic theory of discrete groups, Cambridge Univ. Press, Cambridge, 1989. MR1041575 (91i:58104)

[14] G. Piranian and W. J. Thron, Convergence properties of sequences of linear fractional transformations, Michigan Math. J. 4 (1957), 129-135. MR0093578 (20:102)

[15] J. G. Ratcliffe, Foundations of hyperbolic manifolds, Springer, New York, 1994. MR1299730 (95j:57011)

[16] C. A. Rogers, Hausdorff measures, reprint of the 1970 original, Cambridge Univ. Press, Cambridge, 1998. MR.1692618 (2000b:28009)

[17] B.-W. Wang and J. Wu, Hausdorff dimension of certain sets arising in continued fraction expansions, Adv. Math. 218 (2008), no. 5, 1319-1339. MR.2419924 (2009d:11115)

Department of Mathematics and Statistics, The Open University, Milton Keynes MK7 6AA, UNITED KINGDOM

E-mail address: i.short@open.ac.uk 